\title{
A Rare Case of IgG4 Related Disease Masquerading as Meningioma
}

\author{
Binsu Ann Benny, Suresh Kumar S, Harikrishnan V
}

\section{ABSTRACT}

Rationale: IgG4-related disease (IgG4 RD) is a fibro-inflammatory condition that can affect any organ and can lead to the formation of pseudo tumoral lesions requiring differential diagnosis with various malignancies. IgG4 RD can rarely present as an intracranial space occupying lesion. We are reporting a rare case of central nervous system IgG4 RD which presented as seizures with mass lesion.

Patient Concerns: A 78-year-old man with bronchial asthma and type 2 diabetes mellitus, well controlled on bronchodilators and oral hypoglycemic agents presented with recurring seizures for 1 month. He gave history of a fall and had sustained a wound over the right forehead. At presentation, he was conscious, oriented, and didn't have any focal neurological deficits.

Diagnosis: Due to the recurring seizures and fall, a contrast enhanced MRI brain was done and was reported as a right temporal space occupying lesion in the lateral sphenoid wing. Meningioma was considered as a possibility and right frontotemporal parietal craniotomy and excision was performed. However, biopsies revealed characteristic findings of IgG4-related disease, including 1) dense lymphoplasmacytic infiltration, 2) fibrosis arranged at least focally in a storiform pattern, and 3) obliterative phlebitis, which confirmed the diagnosis. His IgG4 levels were normal $0.58 \mathrm{~g} / \mathrm{L}$ [normal range: $0.03-2.01 \mathrm{~g} / \mathrm{L}]$.

Intervention: Patient underwent right frontotemporal parietal craniotomy and excision of mass under general anesthesia. Histopathology was suggestive of IgG4 RD and the patient was started on immunosuppressive therapy including dexamethasone and mycophenolate mofetil. Antiepileptic medications were continued, and he responded to treatment.

Keywords: IgG4- related disease, Meningioma, IgG4 levels, CNS space occupying lesion.

\section{INTRODUCTION}

IgG4-related diseases (IgG4RD) are a group of disorders defined by high serum IgG4 concentrations and tissue infiltration by IgG4-positive plasma cells that can affect a number of organs [1]. IgG4-RD generally strikes people in their forties and fifties, with symptoms appearing between the ages of 50 and 70 although rarely pediatric cases have been reported. With a M:F ratio of 3:7, most studies reveal a general concern for the male sex, particularly for IgG4related pancreatitis. Females, on the other hand, may be more susceptible to IgG4-related sialadenitis and dacryoadenitis. This condition has now been described in virtually every organ system: the biliary tree, salivary glands, periorbital tissues, kidneys, lungs, lymph nodes, meninges, aorta, breast, prostate, thyroid, pericardium, and skin [2]. Patients with autoimmune pancreatitis (AIP), Mikulicz's illness, hypophysitis, Riedel thyroiditis, interstitial pneumonitis, interstitial nephritis, prostatitis, lymphadenopathy, retroperitoneal fibrosis, inflammatory aortic aneurysm, and
Submitted : December 26, 2021

Published : February 1, 2022

ISSN: 2593-8339

DOI: $10.24018 /$ ejmed.2022.4.1.1182

\section{B. Ann Benny}

Amala Institute of Medical Sciences, India.

(e-mail: binsuannbenny@gmail.com)

S. Kumar S

Amala Institute of Medical Sciences, India.

(e-mail: neurosks@gmail.com)

H. V

Amala Institute of Medical Sciences, India.

(e-mail: drharikrishnan86@gmail.com)

*Corresponding Author

inflammatory pseudotumor are among those who have IgG4RD. Because of non-specific radiological findings mimic more prevalent infiltrative disorders such as sarcoidosis, granulomatous disease, tuberculosis, and lymphoma, makes the diagnosis of intracranial IgG4-RD often difficult [3]. Our case report adds to the growing literature of intracranial IgG4 RD by documenting the clinical and radiological findings of our patient who presented to our hospital with intracranial involvement [3].

\section{CASE Report}

A 78-year-old male with well-controlled diabetes mellitus and bronchial asthma presented to our hospital's neurosurgery outpatient department (OPD) with complaints of generalized tonic-clonic seizures, loss of consciousness, headache. Examination showed a wound on the right side of his forehead associated with a history of a fall at home 1 month back. Magnetic Resonance Imaging brain was taken and was reported as right temporal space occupying lesion in the 
lateral sphenoid wing measuring about $41.3 \times 40 \times 39 \mathrm{~mm}$ in size (Fig. 1). Computed tomography (CT) brain (plain and contrast) was taken and reported. A well-defined near homogeneously enhancing extra axial lesion in right temporal region approximately measuring $4.1 \times 4.1 \times 4.1 \mathrm{~cm}$ with surrounding edema, underlying bone erosion and lysis of right greater wing of sphenoid (Fig 2). He was taken up for surgery and pre-operative work up was done and showed elevated levels of erythrocyte sedimentation rate (ESR). A right frontotemporal parietal craniotomy and excision of mass under general anesthesia was done. The biopsies were sent for histopathological correlation.

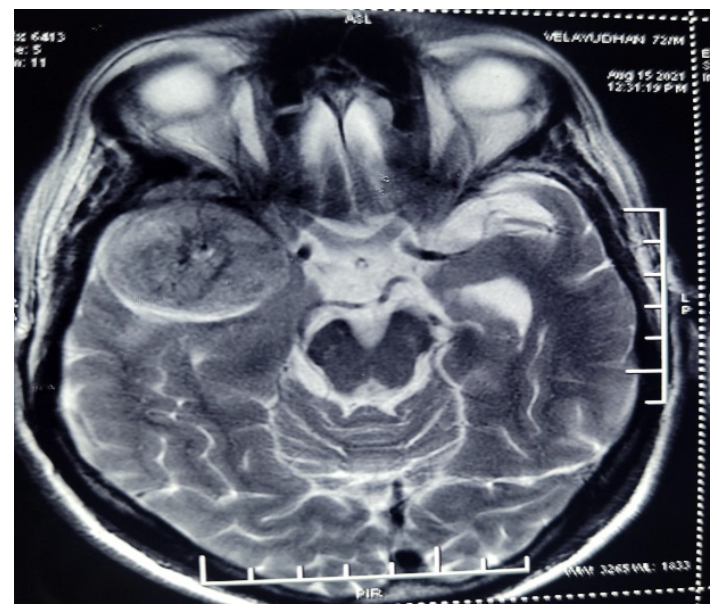

Fig. 1. MRI brain - right temporal space occupying lesion.

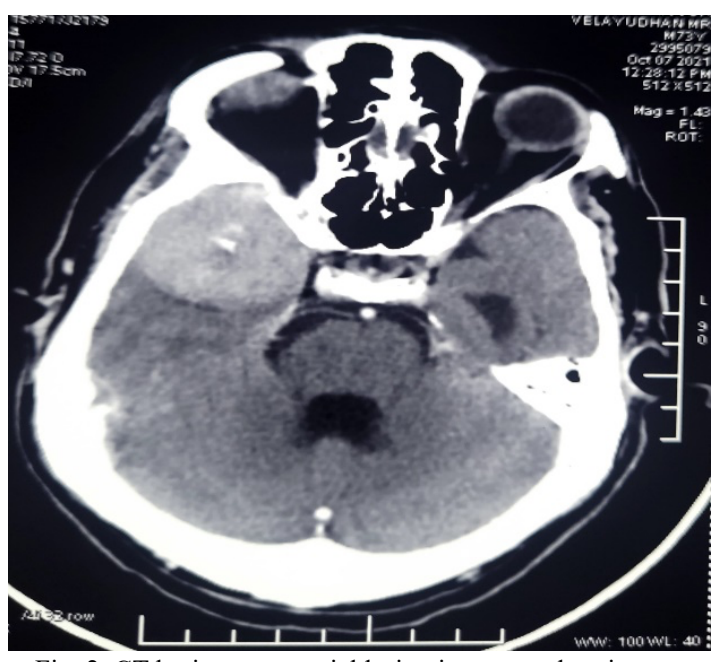

Fig. 2. CT brain - extra axial lesion in temporal region.

\section{A. Histopathological Reported with Microscopy Findings}

Sections showed tissue with dense fibrosis. Some areas showed storiform fibrosis. Blood vessels of varying caliber, lined by endothelial cells and the lumen was obliterated by perivascular concentric fibrosis. Focally lymphoid aggregates were seen forming nodules. The entire tissue was infiltrated by eosinophils in sheets and in areas by sheets of plasma cells and lymphocytes (Fig. 3A, 3B and 3C). Histology was suggestive of $\mathrm{IgG} 4$ related disease.

As the diagnosis of IgG4-RD was confirmed by biopsy, USG bilateral parotids and USG abdomen were done to assess the extent of organ involvement. USG bilateral parotid showed enlarged parotids and US abdomen was normal. Due to financial constraints, patient refused CECT abdomen and chest and PET-CT.

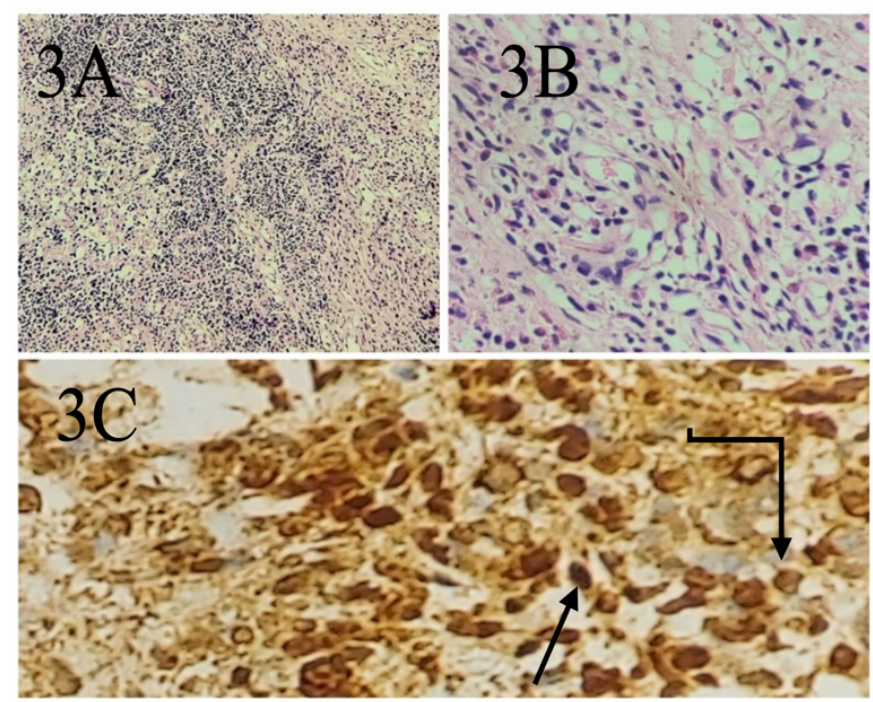

Fig. 3A. A dense lymphoplasmacytic infiltrate. Fig. 3B. Fibrosis has a characteristic "storiform" pattern, typified by a cartwheel appearance of the arranged fibroblasts and inflammatory cells. Fig. 3C. IgG4 immunohistochemical stain highlights many IgG4-positive plasma cells within this single high-powered field.

TABLE I: IMMUNOHISTOCHEMISTRY RESULTS

\begin{tabular}{cc}
\hline \hline BIOMARKERS & RESULT \\
CD138 & $\begin{array}{c}\text { Positive confirming the significant } \\
\text { plasma cell infiltrate. }\end{array}$ \\
IgG4 & $\begin{array}{c}\text { Immunoreactive, highlights } 5-8 \\
\text { plasma cells } / \mathrm{hpf}\end{array}$ \\
IgG & $\begin{array}{c}\text { Immunoreactive, highlights } 35-40 \\
\text { plasma cells } / \mathrm{hpf}\end{array}$ \\
Serum IgG4 levels & $0.58 \mathrm{~g} / \mathrm{L}$ [normal range: $0.03-2.01]$ \\
\hline \hline
\end{tabular}

\section{DISCUSSION}

IgG4-related disease (IgG4-RD) is a fibroinflammatory chronic condition that affects a variety of organs. The presence of numerous IgG4-expressing plasma cells in the tissue and elevated serum IgG4 concentrations are crucial diagnostic criteria of this autoimmune illness [4]. The diagnosis of IgG4-RD is based on three histological criteria on the pathology of IgG4-related disease: dense lymphoplasmacytic infiltration, fibrosis arranged at least focally in a storiform pattern, and obliterative phlebitis [5]. The diagnostic criteria have been explained in Table II. Paradoxically, about $40 \%$ of patients with IgG4-RD who have had a biopsy have normal serum IgG4 levels. Elevated blood IgG4 levels are not required to diagnose IgG4-related inflammatory pseudo-tumor [6]. As our patient's serum IgG4 level was normal, sclerosing fibrosis accompanied with lymphocytes and IgG4-positive plasma cells invading the tissues led to the diagnosis of IgG4-related pseudo-tumor. Other radiological imaging was done, including a neck ultrasound, which revealed enlarged parotids, which is another supportive feature of IgG4 RD.

Recurrent seizures, headache, and loss of consciousness in index patient required CNS imaging and MRI and CT brain reported SOL, possibly meningioma. For diagnosis and 
treatment, surgical excision was done, and biopsy showed characteristic histopathological features of IgG4 RD which was an unexpected diagnosis.

\section{TABLE II: DIAGNOSTIC CRITERIA}

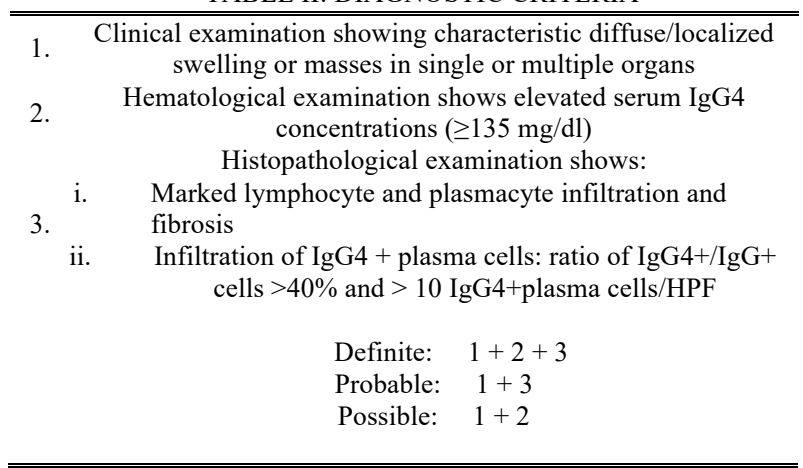

Although histopathological examination is required for a conclusive diagnosis of IgG4-related disease, radiologic imaging plays an important role in demonstrating characteristics suggestive of the diagnosis. A CT scan of the chest, abdomen, and pelvis should be included in the imaging workup for IgG4-related disease to detect suspected multiorgan involvement.

PET-CT (positron emission tomography-computed tomography) is highly recommended by many people for evaluation of IgG4 related disease. Studies have shown that it is useful for diagnosis, extent of organ involvement and response tracking. This imaging method has produced better results when compared to ultrasonography and CT [5].

He was subsequently started on prednisolone $1 \mathrm{mg} / \mathrm{kg}$ and mycophenolate mofetil as a steroid sparing immunosuppressive therapy. He responded well and is currently symptomatically better with no focal neurological deficits.

\section{CONCLUSIONS}

Diagnosis of IgG4 RD will be based on radiological imaging and biopsy showing characteristic findings of IgG4 RD. Glucocorticoids and other immunosuppressive medications like rituximab, mycophenolate mofetil, azathioprine are treatment options for IgG4-related inflammatory pseudo tumors. Local radiation therapy has been demonstrated to be useful in some circumstances when patients are not steroid responsive. As a result, future advancements in the treatment of IgG4-RD are more likely to emerge in the form of novel parameters such as plasma blasts, which could allow for disease activity monitoring and precise retreatment timing in order to avoid relapses before they occur [5].

\section{AUTHORS CONTRIBUTION}

1) Conceptualization - Dr. Binsu Ann Benny (Neurosurgery).

2) Supervision - Dr. Suresh Kumar S (HOD - Dept. of Neurosurgery) and Dr. Harikrishnan V (Assistant Professor - Dept. of Clinical Immunology and Rheumatology).
3) Writing original draft - Dr. Binsu Ann Benny (Neurosurgery).

4) Writing- review and editing -Dr. Binsu Ann Benny, Dr. Suresh Kumar S, Dr. Harikrishnan V.

\section{REFERENCES}

[1] Goulam-Houssein S, Grenville JL, Mastrocostas K, Munoz DG, Lin A Bharatha A, et al. IgG4-related intracranial disease. Neuroradiol J. 2019; 32(1): 29-35.

[2] Deshpande V, Zen Y, Chan JK, Yi EE, Sato Y, Yoshino T, et al. Consensus statement on the pathology of IgG4-related disease. Mod Pathol. 2012; 25(9): 1181-92.

[3] Perugino CA, AlSalem SB, Mattoo H, Della-Torre E, Mahajan V, Ganesh G, et al. Identification of galectin-3 as an autoantigen in patients with IgG4-related disease. J Allergy Clin Immunol. 2019; 143(2): 736-745.e6.

[4] Vidarsson G, Dekkers G, Rispens T. IgG subclasses and allotypes: from structure to effector functions. Front Immunol. 2014; 5: 520.

[5] Kamisawa T, Okazaki K. Diagnosis and treatment of IgG4-related disease. Curr Top Microbiol Immunol. 2017; 401: 19-33.

[6] Liu X, Wang R, Li M, Chen G. IgG4-related inflammatory pseudotumor involving the clivus: A case report and literature review. Front Endocrinol. 2021; 12: 666791. 